\title{
Direct Observation of Double Hydrogen Transfer via Quantum Tunneling in a Single Porphycene Molecule on a Ag(110) Surface
}

\author{
Matthias Koch, ${ }^{\dagger}$ Mark Pagan, ${ }^{\ddagger}$ Mats Persson, ${ }^{\ddagger}$ Sylwester Gawinkowski, ${ }^{\S}$ Jacek Waluk, ${ }^{\S, \|}$ \\ and Takashi Kumagai* ${ }^{\dagger}+0$
}

\author{
${ }^{\dagger}$ Department of Physical Chemistry, Fritz-Haber Institute of the Max-Planck Society, Faradayweg 4-6, Berlin 14195, Germany \\ ${ }^{\ddagger}$ Surface Science Research Centre and Department of Chemistry, University of Liverpool, Liverpool L69 3BX, United Kingdom \\ ${ }^{\S}$ Institute of Physical Chemistry, Polish Academy of Sciences, Kasprzaka 44/52, Warsaw 01-224, Poland \\ "Faculty of Mathematics and Natural Sciences, College of Science, Cardinal Stefan Wyszyński University, Dewajtis 5, Warsaw 01-815, \\ Poland
}

\section{Supporting Information}

ABSTRACT: Quantum tunneling of hydrogen atoms (or protons) plays a crucial role in many chemical and biological reactions. Although tunneling of a single particle has been examined extensively in various one-dimensional potentials, many-particle tunneling in high-dimensional potential energy surfaces remains poorly understood. Here we present a direct observation of a double hydrogen atom transfer (tautomerization) within a single porphycene molecule on a $\operatorname{Ag}(110)$ surface using a cryogenic scanning tunneling microscope (STM). The tautomerization rates are temperature independent below $\sim 10$ $\mathrm{K}$, and a large kinetic isotope effect (KIE) is observed upon substituting the transferred hydrogen atoms by deuterium, indicating that the process is governed by tunneling. The observed KIE for three isotopologues and density functional theory calculations reveal that a stepwise transfer mechanism is dominant in the tautomerization. It is also found that the tautomerization rate is increased by vibrational excitation via an inelastic electron tunneling process. Moreover, the STM tip can be used to manipulate the tunneling dynamics through modification of the potential landscape.

\section{Double hydrogen-atom transfer via quantum tunneling}
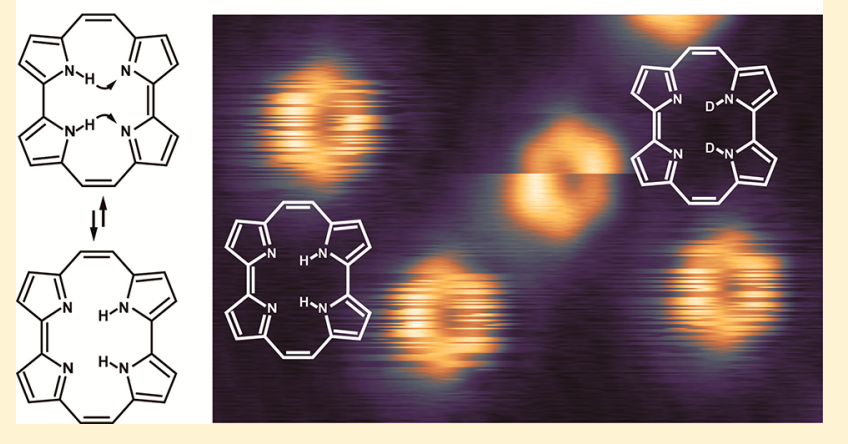

\section{INTRODUCTION}

Hydrogen-transfer reactions are involved in a variety of chemical and biological processes. ${ }^{1}$ As being the lightest element, the hydrogen atom is subject to important nuclear quantum effects (NQEs), ${ }^{2-5}$ leading to peculiar static and dynamic properties which cannot be rationalized by classical mechanics. For instance, already in 1927 the importance of quantum tunneling was pointed out by Hund for an intramolecular hydrogen rearrangement such as flipping of ammonia (umbrella motion). ${ }^{6}$ Tunneling is not only of fundamental interest in quantum physics/chemistry but also plays a crucial role in important chemical ${ }^{7,8}$ and enzymatic ${ }^{9}$ reactions. Although single-particle tunneling has been well studied in various types of one-dimensional potentials, ${ }^{10}$ the accurate description of many-particle tunneling in a multidimensional potential energy surface is far from complete. Additionally, intermolecular interactions or crystal fields could affect the tunneling dynamics in condensed phases, but it remains a challenging task to probe directly NQEs at the singlemolecule level. Recently, low-temperature STM has been proven to be a novel and powerful tool to investigate NQEs of hydrogen on surfaces in real space, whereby hydrogen diffusion, ${ }^{11}$ hydrogen-bond rearrangement in small water clusters $^{12,15}$ and flipping motion of hydrogen atom ${ }^{14}$ via tunneling, and the zero-point energy contribution in the hydrogen bond ${ }^{13,16}$ were directly observed. Additionally, theoretical calculations also highlighted considerable impacts of NQEs on the structure and dynamics of adsorbed molecules. ${ }^{17-19}$ Interestingly, tunneling was found to be an important contribution to hopping (diffusion) of heavy atoms and molecules such as $\mathrm{Cu}^{20} \mathrm{Co}^{21}$ and $\mathrm{CO}^{22}$ on a $\mathrm{Cu}(111)$ surface at cryogenic temperatures.

In a multiple hydrogen (proton) transfer, the process occurs either in a stepwise or in a concerted fashion. In the former case, individual hydrogen atoms move separately and there exist multiple transition states and metastable intermediate structures along the reaction pathway. In the latter case, the hydrogen atoms are transferred collectively and the reaction coordinate exhibits a single transition structure. Tautomerizations in porphyrins and phthalocyanines have served as fascinating models to study double hydrogen transfer

Received: July 3, 2017

Published: August 21, 2017 

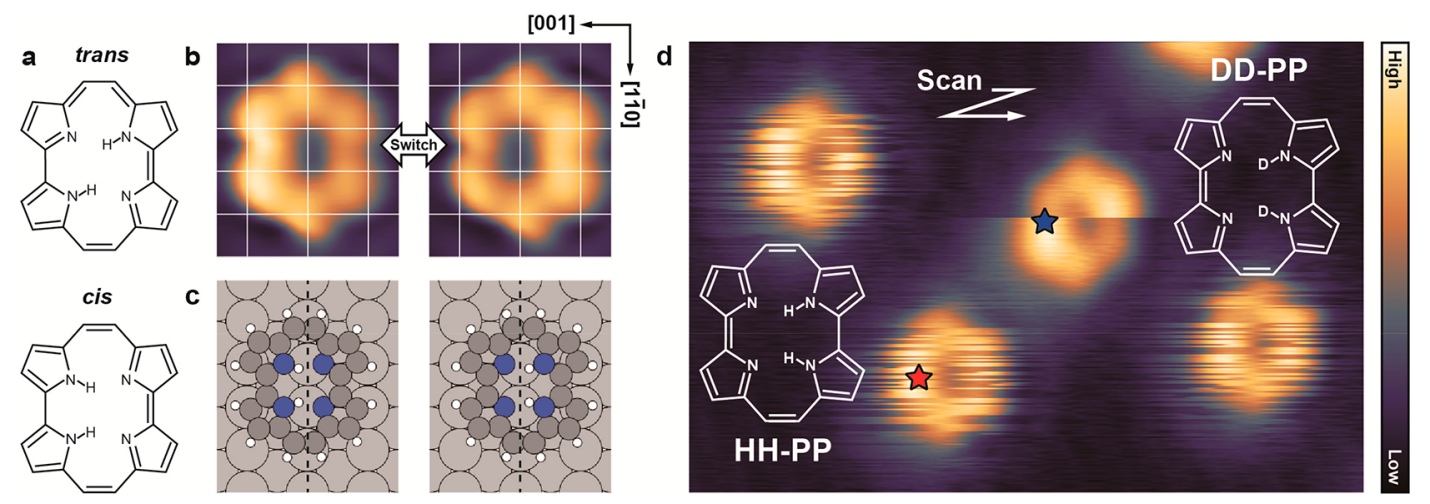

Figure 1. Porphycene molecule on $\mathrm{Ag}(110)$. (a) Two tautomeric forms of porphycene. (b) STM images of a single DD-porphycene molecule (5 K, $V_{\text {bias }}=50 \mathrm{mV}, I_{\mathrm{t}}=0.1 \mathrm{nA}$, size $\left.1.6 \times 2.0 \mathrm{~nm}^{2}\right)$. White grid lines represent the surface lattice of $\mathrm{Ag}(110)$. (c) Top view images of calculated cis structure and its mirror reflection. Dashed lines represent the high-symmetry (mirror) axis of the surface. (d) STM image of HH- and DDporphycene molecules $\left(5 \mathrm{~K}, V_{\text {bias }}=50 \mathrm{mV}, I_{\mathrm{t}}=0.05 \mathrm{nA}\right.$, size $\left.4.0 \times 6.0 \mathrm{~nm}^{2}\right)$. Scanning direction is indicated by the white arrow. This raster scanning results in discrete changes of the STM image when porphycene tautomerizes.

reactions. ${ }^{1}$ Recently, low-temperature STM has been employed to investigate tautomerization in a single molecule adsorbed on surfaces. $^{23-25}$ These pioneering studies proposed that tautomerization could be exploited to make a single-molecule switching device in future molecular electronics. Porphycene, the first synthesized constitutional isomer of porphyrin, ${ }^{26}$ has emerged as an intriguing model for intramolecular hydrogen bonding and a double hydrogen transfer. ${ }^{27,28}$ Because of the strong intramolecular hydrogen bonds, the potential landscape of tautomerization in porphycene is significantly shallower as compared to porphyrins and phthalocyanines, leading to pronounced NQEs, that is, hydrogen tunneling. ${ }^{29}$ Porphycene occurs as the trans or cis tautomer (Figure 1a) with comparable total energies within only a few $\mathrm{kcal} / \mathrm{mol}$ (ref 30 and see our calculated results in Supporting Information). In the gas phase, trans-trans tautomerization takes place via coherent tunneling and its rate was estimated to be $6 \times 10^{11} \mathrm{~Hz}$ from the tunneling splitting $\left(4.4 \mathrm{~cm}^{-1}\right)$ in the vibronic levels at the ground state. ${ }^{31}$ A crucial role of tunneling, even at room temperature, was also observed for porphycene in the condensed phase. ${ }^{32}$ Because the barrier heights of the stepwise and concerted pathway were also estimated to be comparable, it was proposed that the tautomerization might take place in a competing manner. ${ }^{33}$ Additionally, the tautomerization coordinate of porphycene is essentially multidimensional, and several vibrational modes act as a reaction promoter or inhibitor through anharmonic intermode coupling. ${ }^{31}$ However, the tunneling process was found to be quenched upon adsorption on a $\mathrm{Cu}(110)$ surface due to the interaction of the porphycene with the surface atoms. $^{34,35}$ Here we present that tautomerization of porphycene occurs via tunneling on a less reactive $\mathrm{Ag}(110)$ surface and elucidate the reaction mechanism by a combination of lowtemperature STM experiments and density functional theory (DFT) calculations.

\section{METHODS}

Experiments were carried out under ultrahigh vacuum conditions $(<2$ $\times 10^{-10} \mathrm{mbar}$ ) with a low-temperature STM from Omicron Nanotechnology $\mathrm{GmbH}$ and operated with a Nanonis Controller System from Specs $\mathrm{GmbH}$. An electrochemically etched Au tip was used as a STM probe. The bias voltage $\left(V_{\text {bias }}\right)$ was applied to the sample, and the tip was grounded. A single-crystalline $\operatorname{Ag}(110)$ surface was cleaned by repeated cycles of argon ion sputtering and annealing to $670 \mathrm{~K}$. We investigated three isotopologues, namely, $\mathrm{HH}$-, $\mathrm{HD}$-, and
DD-porphycene. In the latter two species, the inner hydrogen atoms within the molecular cavity are replaced by one or two deuterium atoms. The deuteration was carried out by repeated recrystallization cycles with $\mathrm{CD}_{3} \mathrm{OD} / \mathrm{CD}_{2} \mathrm{Cl}_{2}$ solution. ${ }^{36}$ The partially deuterated species, HD-porphycene, is involved as an impurity in the deuteration process of the parent $\mathrm{HH}$-porphycene, and its existence was established by high-resolution electronic spectroscopy with ultracold molecules isolated in helium nanodroplets. ${ }^{31}$ We used a deuterated sample that contains HD-species. The purity of the sample used was checked under UHV conditions by depositing the sample onto a $\mathrm{Cu}(110)$ surface where each species can be discriminated by singlemolecule vibrational spectroscopy of the STM (see Supporting Information for details of the identification). Porphycene molecules were evaporated from a Knudsen cell at $\sim 450 \mathrm{~K}$, while the $\mathrm{Ag}(110)$ sample was kept at room temperature.

The geometric structures and the minimum energy paths (MEPs) of the adsorbed porphycene on the $\mathrm{Ag}(110)$ surface were obtained from periodic, plane-wave DFT calculations using the Vienna ab initio simulation program (VASP). ${ }^{37}$ The electron-ion core interactions and the exchange-correlation effects were treated using the Projector Augmented Wave (PAW) method ${ }^{38}$ and the optB86B version of the van der Waals density functional, ${ }^{39-42}$ respectively. The robustness of the calculated results was investigated using the BEEF-vdW ${ }^{43}$ and the vdW-DF-cx functionals. ${ }^{44}$ The $\mathrm{Ag}(110)$ surface was represented in a supercell by a four-layer slab with a $4 \times 6$ surface unit cell and a $20 \AA$ vacuum region. The MEPs were computed with the nudged elastic band method. ${ }^{45,46}$ Further details of the calculations can be found in the Supporting Information.

\section{RESULTS AND DISCUSSION}

Figure $1 \mathrm{~b}$ displays topographic STM images of a single porphycene molecule on $\operatorname{Ag}(110)$ at $5 \mathrm{~K}$. The crescent-shaped appearance is similar to that on $\mathrm{Cu}(110)^{35}$ and indicates that the porphycene adsorbs in the cis configuration. Our DFT calculations also predict the cis configuration to be the most stable tautomer on $\operatorname{Ag}(110)$ (Figure 1c), although the relative energy difference $\Delta E_{\text {cis-trans }}$ is quite small $(0.023 \mathrm{eV})$. The brighter side in the topographic image of the porphycene (Figure 1b) corresponds to the side where two hydrogen atoms are bonded to the pyrrole nitrogen atoms. ${ }^{35}$ In the gas phase, solution, or crystalline state, the trans configuration has been observed to be the most stable tautomer. However, the preference of the cis configuration on the $\operatorname{Ag}(110)$ surface could be explained by a relatively strong interaction between the amine nitrogen atoms (with a lone pair) in the molecular cavity and the surface atoms underneath. The adsorption also 

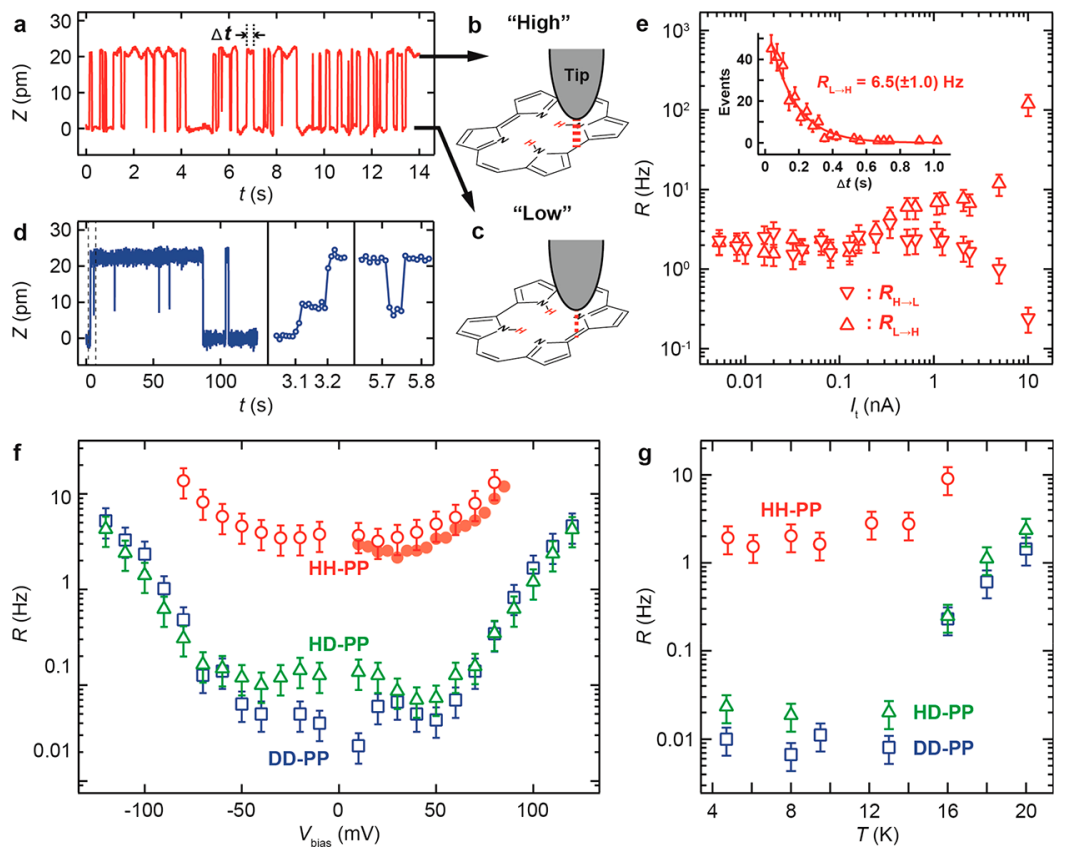

Figure 2. Dependence of tautomerization rate on tunneling current, bias voltage, and temperature. (a) Trace of tip height $(Z)$ measured for HHporphycene. Traces are acquired at a set current $\left(I_{\mathrm{t}}\right)$ of $5 \mathrm{pA}$ at $V_{\text {bias }}=30 \mathrm{mV}$. Lateral tip position during the measurement is indicated by the red and blue stars in Figure 1d. (b and c) Schematic of "high" and "low" states. (d) Trace of tip height $(Z)$ measured for DD-porphycene. Right panels show magnified $Z$ traces in the region marked by the dashed lines in the left, where a short-lived trans state is observed. (e) Current $\left(I_{\mathrm{t}}\right)$ dependence of the tautomerization rates for the "high" to "low" transition $\left(R_{\mathrm{H} \rightarrow \mathrm{L}}\right)$ and the opposite process $\left(R_{\mathrm{L} \rightarrow \mathrm{H}}\right)$ for HH-porphycene measured at $5 \mathrm{~K}$. $V_{\text {bias }}$ is fixed to $20 \mathrm{mV}$ during the measurement. Inset shows a histogram of the dwell time in the tip height trace (interval between tautomerization events, $\Delta t$ ) for the "low" to "high" process and measured at $I_{\mathrm{t}}=0.63 \mathrm{nA}$. The rate is determined to be $6.5( \pm 1.0) \mathrm{Hz}$ by fitting the data to an exponential decay function. (f) Voltage dependence of the tautomerization rate for $\mathrm{HH}-, \mathrm{HD}$-, and DD-porphycene measured at $5 \mathrm{~K}$ with $I_{\mathrm{t}}=5 \mathrm{pA}$. Open markers represent the data that are obtained under the same tip conditions. Filled circles are the data obtained with a different tip. $(\mathrm{g}) \mathrm{Temperature}(T)$ dependence of the tautomerization rate for HH-, HD-, and DD-porphycene measured at $I_{\mathrm{t}}=10 \mathrm{pA}$ and $V_{\text {bias }}=10 \mathrm{mV}$.

causes a slight deformation of the porphycene macrocycle that is planar in the gas phase. Our calculations unveil that this deformation makes the trans configuration even more preferable than the cis configuration in the absence of the surface (see Supporting Information). Thus, the stabilization of the cis tautomer on the surface is not caused by the buckling but is caused by the molecule-surface interaction.

It is found that the porphycene switches between two orientations as shown in Figure $1 \mathrm{~b}$, which corresponds to the cis $\leftrightarrow$ cis tautomerization through a double hydrogen transfer within the molecular cavity. Interestingly, this reaction occurs spontaneously even at $5 \mathrm{~K}$ and exhibits a strong isotope effect. Figure 1d shows an STM image of normal and deuterated species (HH- and DD-porphycene, respectively). The rapid tautomerization rate of $\mathrm{HH}$-porphycene is reflected by its fluctuating image, whereas the rate is much smaller for DDporphycene, and only a single tautomerization event is observed in Figure 1d, thus revealing a large kinetic isotope effect (KIE). Additionally, we find that the cis $\leftrightarrow$ cis tautomerization results in a small displacement of the molecule along the [001] direction (Figure 1b), which is also confirmed by the calculated structures (Figure 1c). This displacement is clearly visible in the STM images of the tautomerization of DDporphycene in Figure 1d.

The observed KIE indicates the dominant contribution of tunneling to tautomerization, as is further confirmed by investigating the dependence of the tautomerization rate on the tunneling current, the bias voltage, and the temperature. Tautomerization of an individual porphycene was directly monitored by tracing the change in the tip height over the molecule (Figure 2a) while fixing the lateral tip position as indicated by the markers in Figure 1d. The tautomerization results in a random telegraph signal where the "high" and "low" states correspond to the configurations such that the inner hydrogen atoms locate close to and away from the tip, respectively (see schematics in Figure $2 b$ and $2 c$ ). The change in the tip height of about $0.2 \AA$ corresponds to the difference between the topographic heights of the brighter and the darker side of porphycene which correspond to the pyrrole rings with imine and amine nitrogen atoms, respectively. For DDporphycene an additional level (a third state) can be observed in the tip height trace, which appears intermittently in between the "high" and the "low" states (Figure 2d). This state should be attributed to the metastable trans configuration (as discussed below). However, this trans state was not observed for $\mathrm{HH}$ porphycene most probably due to the larger tunneling rates of hydrogen than deuterium atoms, resulting in a shorter lifetime of the trans state for $\mathrm{HH}$-porphycene. This makes it more difficult to capture the trans state of HH-porphycene than for DD-porphycene by the limited time resolution of the STM (a few milliseconds in the measurement used). In order to analyze the tautomerization in a quantitative manner, the reaction rate is extracted from the tip height trace (Figure $2 \mathrm{a}$ and $2 \mathrm{~d}$ ). Two different ways were used to obtain the rate; fitting the histogram of the dwell time (interval between tautomerization events) to an exponential decay function (as shown in the inset of Figure 2e) or dividing the total number of events by the total trace time. We confirmed that both give similar results for $\mathrm{HH}$ porphycene. The latter way was used for $\mathrm{HD}$ - and $\mathrm{DD}$ porphycene because of much less tautomerization events which 
were not sufficient to conduct the histogram analysis. Figure $2 \mathrm{e}$ shows the current dependence of the tautomerization rate for $\mathrm{HH}$-porphycene at a fixed bias voltage $\left(R_{\mathrm{H} \rightarrow \mathrm{L}}\right.$ and $R_{\mathrm{L} \rightarrow \mathrm{H}}$ denote the rate from "high" to "low" transition and the opposite process, respectively). $R_{\mathrm{H} \rightarrow \mathrm{L}}$ and $R_{\mathrm{L} \rightarrow \mathrm{H}}$ show a rather weak dependence on the current below $\sim 0.2 \mathrm{nA}$, indicating that tunneling electrons, an electric field, and the tip-molecule interaction do not play a significant role (the similar behavior was also confirmed for the other isotopologues, see Supporting Information). However, $R_{\mathrm{H} \rightarrow \mathrm{L}}$ and $R_{\mathrm{L} \rightarrow \mathrm{H}}$ clearly deviate from each other at higher currents, which should be ascribed to the deformation of the tautomerization potential energy surface, caused by the tip-molecule interaction upon tip approach (cf. Figure 4). Figure $2 \mathrm{f}$ shows the bias voltage dependence of the tautomerization rate measured with the tunneling current fixed to $5 \mathrm{pA}$. Because $R_{\mathrm{H} \rightarrow \mathrm{L}}$ and $R_{\mathrm{L} \rightarrow \mathrm{H}}$ are nearly equal at low tunneling currents (Figure 2e), the rates in Figure $2 \mathrm{f}$ are determined from the dwell time analysis without discriminating the two processes (same applies to Figure $2 \mathrm{~g}$ ). The tautomerization rate rapidly increases above $50 \mathrm{mV}$ for all isotopologues. At lower voltages, the rate shows a slight increase, which should be attributed to the proximity of the tip and the molecule. The voltage-dependent rate appears symmetric at both bias polarities, and the onsets of the rate increase are similar for all isotopologues. The HOMO-LUMO gap of porphycene is about $2 \mathrm{eV},{ }^{29}$ which does not match the energy of the threshold voltage $(\sim 50 \mathrm{mV})$. Therefore, the increase of the tautomerization cannot be attributed to the onset of such an electronic excitation. However, it is likely that the threshold voltage represents vibrational excitation of a skeletal mode of porphycene which could couple with the tautomerization coordinate. ${ }^{31}$ Hence, we conclude that the increase of the rate occurs by vibrational excitation via an inelastic electron tunneling process. ${ }^{34}$ In Figure $2 \mathrm{f}$, the voltage dependence for the three isotopologues (open markers) was obtained under the same tip conditions. However, the rate shows a variation when it is measured under different tip conditions, e.g., use of a different tip or execution of in situ tip preparations like application of a voltage pulse or tip indentation into the surface. As shown by the filled circles in Figure $2 \mathrm{f}$, a different tip resulted in a small decrease (or increase with another tip) in the tautomerization rate, but the overall behavior of the current, voltage, and temperature dependence was unchanged. The error bar of the rate in all presented data takes into account this experimental uncertainty.

The cis $\leftrightarrow$ cis tautomerization via tunneling on $\operatorname{Ag}(110)$ is conclusive from the temperature-dependent rates (Figure $2 \mathrm{~g}$ ). For all isotopologues, the rate is independent of the temperature below $\sim 10 \mathrm{~K}$ and exhibits a large KIE of about 100 between $\mathrm{HH}$ - and DD-porphycene, while the KIE between DD- and HD-porphycene is about 2. The Bell-Limbach tunneling model ${ }^{47}$ - an accepted model to explain a temperature-dependent reaction rate of multiple hydrogen (proton) transfers including tunneling at low temperatures-revealed that the KIE of the rate constant, $k_{\mathrm{LL}}(\mathrm{L}=\mathrm{H}$ or D), in the tunneling regime to be $k_{\mathrm{HH}} \gg k_{\mathrm{HD}} \cong 2 k_{\mathrm{DD}}$ or $k_{\mathrm{HH}} / k_{\mathrm{HD}} \approx k_{\mathrm{HD}} /$ $k_{\mathrm{DD}}$ for the stepwise or concerted mechanisms, respectively. ${ }^{48-51}$ The theoretical calculations by Smedarchina and co-workers ${ }^{52}$ corroborated these ratios of KIEs for the stepwise and concerted mechanisms for tautomerization of porphycene isotopologues using a quantum mechanical model with an approximate instanton method. In the stepwise mechanism, $k_{\mathrm{HD}}$ is expected to be close to $k_{\mathrm{DD}}$ because the deuterium transfer should be the rate-limiting step in the HD species. On the other hand, $k_{\mathrm{HD}}$ should be closer to $k_{\mathrm{HH}}$ in the concerted mechanism than for the stepwise one. Our result is in line with the stepwise scenario, and we assign the third state observed in the tip height trance for DD-porphycene (Figure 2d) to the metastable trans configuration.

The reaction pathway and barrier are examined using DFT calculations. It is revealed that the stepwise mechanism (Figure $3 a)$ via the first-order saddle points and the metastable trans

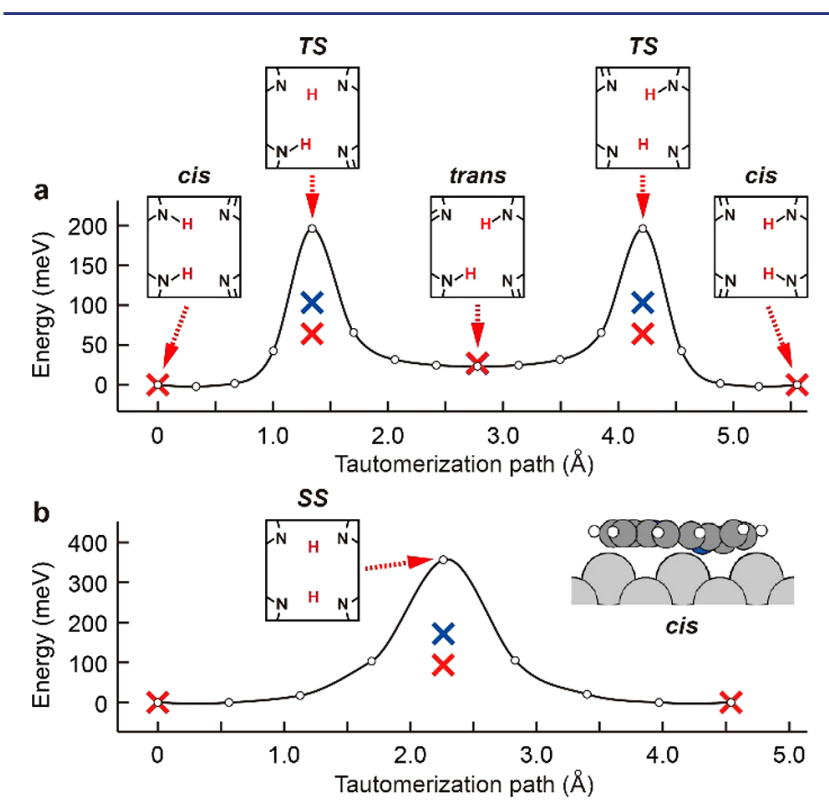

Figure 3. Minimum energy paths (MEPs) of porphycene on $\mathrm{Ag}(110)$. ( $a$ and $b$ ) Calculated stepwise and concerted MEPs, respectively. Red and blue crosses represent the zero-point corrected energy for $\mathrm{HH}$ and DD-porphycene, respectively. TS: first-order transition state. SS: second-order saddle point. Inset in b shows a side view of the calculated cis structure.

configuration shows a lower barrier than that of the concerted mechanism (Figure $3 \mathrm{~b}$ ) via the second-order saddle point. The zero-point energy (ZPE) corrected barrier is calculated to be $0.064(0.103)$ and $0.094(0.172) \mathrm{eV}$ for the stepwise and concerted pathway, respectively (values within parentheses refer to DD-porphycene). In either case, the barrier cannot be overcome through thermal activation at $5 \mathrm{~K}$, corroborating that the reaction is dominated by tunneling. These calculated barrier heights seem to suggest that the stepwise pathway is more likely than the concerted one, which is consistent with experiment. Meanwhile, the barrier width (tunneling path length) also influences the tunneling process. However, in the case of multidimensional tunneling, it is not straightforward to define and find a tunneling path and consequently to determine the tunneling rate. ${ }^{19}$ For the porphycene on $\operatorname{Ag}(110)$ we found a significant displacement of carbon and nitrogen atoms upon the cis $\leftrightarrow$ cis tautomerization (cf. Figure 1); thus, the tunneling proceeds in a highly multidimensional potential energy surface, which hampers an accurate estimation of the tunneling rate.

The tautomerization rate via tunneling on $\operatorname{Ag}(110)$ is considerably reduced compared to the rate in the gas phase $\left(6 \times 10^{11} \mathrm{~Hz}\right.$ for $\mathrm{HH}$-porphycene $\left.{ }^{31}\right)$. This reduction is explained by an increase of the tautomerization barrier as compared to $0.023( \pm 0.002) \mathrm{eV}$ estimated for the trans $\leftrightarrow$ trans tautomerization in inert media. ${ }^{29}$ Additionally, the symmetry 
lowering of the porphycene upon adsorption may also reduce the tunneling rate through an increase of the tunneling path length, caused by the motion of the porphycene during tautomerization. ${ }^{19}$ This is in contrast to the gas phase where the tautomerization coordinate is dominated by the motion of the inner hydrogen atoms. Moreover, the calculated structure (inset of Figure 3b) reveals a slight deformation of the porphycene macrocycle, resulting from a stronger interaction of the amine nitrogen atoms with the surface $\mathrm{Ag}$ atoms than the imine nitrogen atoms. This molecule-surface interaction stabilizes the cis configuration over the trans configuration but gives rise to an increase of the tautomerization barrier. It should be noted that no spontaneous tautomerization (via tunneling) was observed for the porphycene on $\mathrm{Cu}(110)$ at $5 \mathrm{~K}$, although tautomerization can be induced by the STM through an inelastic electron tunneling process. ${ }^{34,35}$ The absence of the tunneling process can be rationalized by the stronger molecule-surface interaction on $\mathrm{Cu}(110)$ than that on $\mathrm{Ag}(110)$, as implied by a larger deformation of the molecular skeleton on $\mathrm{Cu}(110)$ (see Supporting Information). The tautomerization barrier of $\mathrm{HH}$-porphycene on $\mathrm{Cu}(110)$ is calculated to be 0.20 and $0.35 \mathrm{eV}$ for the stepwise and concerted mechanism, respectively, which are larger than $\operatorname{Ag}(110)$ (see Supporting Information). This increase of the barrier is found to be robust to the choice of exchangecorrelation functional (see Supporting Information).

The local environment of a molecule can strongly influence tunneling dynamics. ${ }^{15} \mathrm{We}$ find that the tunneling rate (Figure $2 \mathrm{e})$ and the population of the "high" and "low" state (Figure 4a and $4 \mathrm{~b}$ ), thus the kinetics of the cis $\leftrightarrow$ cis tautomerization on $\operatorname{Ag}(110)$, can be manipulated by varying the tip-molecule distance. The tautomerization is eventually quenched when the tip is brought close to the molecule. Figure $4 \mathrm{~b}$ shows the populations of the "low" and "high" states obtained under two different tip conditions (the tip apex structure was changed by a controlled indentation of the tip into the surface) and with a CO-modified tip. Although the variation of the rate and population also depends on the tip conditions, the general tendencies remain similar. The increasing population of the "high" state with decreasing tip-molecule distance suggests that an attractive interaction between tip and molecule makes the "high" state more preferable as schematically shown in Figure 4c. Our recent study ${ }^{53}$ has demonstrated that the symmetric potential energy surface of the cis $\leftrightarrow$ cis tautomerization on $\mathrm{Cu}(110)$ can be deformed in the presence of the tip such that the "high" state becomes more favorable than the "low" state in the attractive force regime, whereas their relative stability reverses in the repulsive regime. Because the tip-molecule distance in Figure $4 \mathrm{~b}$ is expected to be relatively large, the interaction stays in the attractive force regime which should lead to preference of the "high" state.

\section{CONCLUSION}

A double hydrogen transfer (cis $\leftrightarrow$ cis tautomerization) via tunneling was observed directly in single porphycene molecules on a $\operatorname{Ag}(110)$ surface using a low-temperature STM. The dominant contribution of tunneling was manifested as a temperature-independent tautomerization rate below $\sim 10 \mathrm{~K}$. Additionally, the short-lived trans state observed for DDporphycene, the isotope ratio of the tautomerization rate between different isotopologues, and the DFT calculations indicated that the tautomerization occurs by the stepwise mechanism rather than the concerted one. The DFT
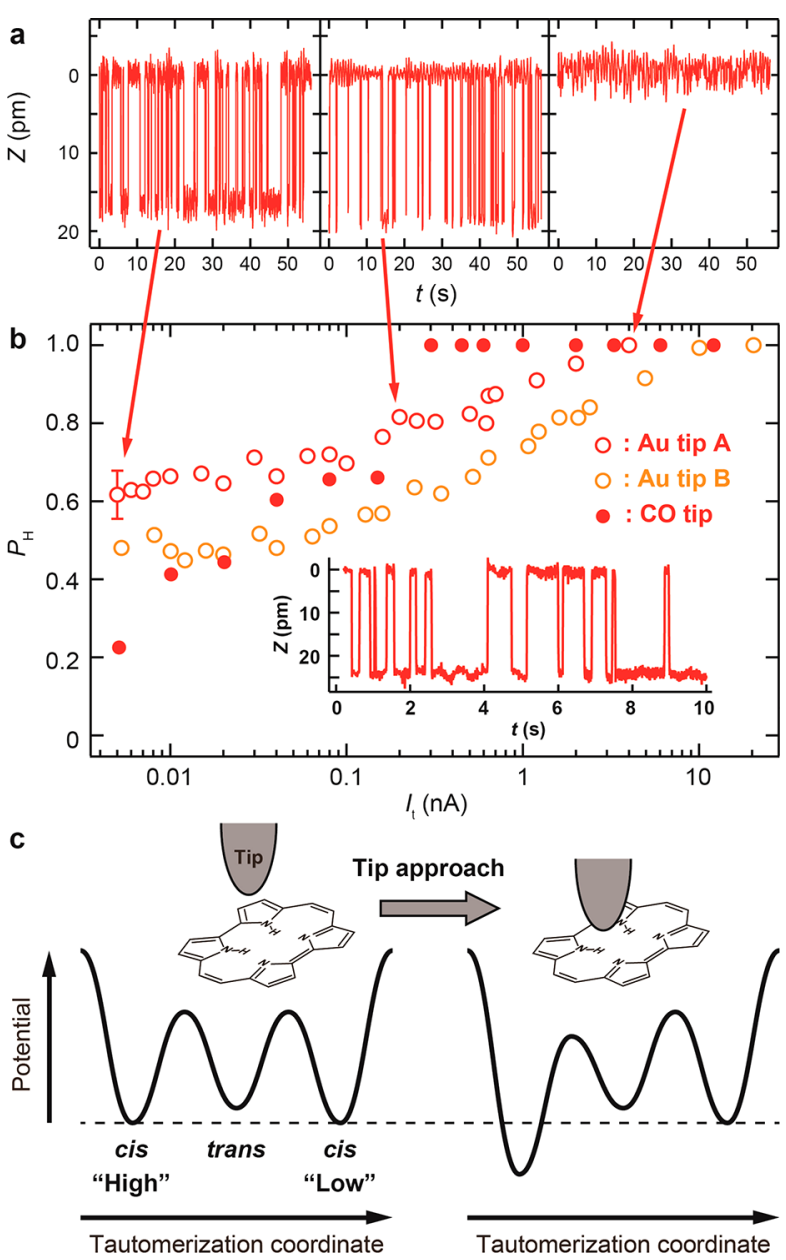

Figure 4. Influence of the tip on the tautomerization dynamics. (a) Tip height traces measured for HH-porphycene at three different gap distances. Lateral tip position is fixed at the position indicated in Figure 1d. (b) Fractional population of the "high" state as a function of a set current (gap distance). Data were obtained for $\mathrm{HH}$-porphycene at $5 \mathrm{~K}$ with different Au tips (open circles, at $V_{\text {bias }}=20 \mathrm{mV}$ ) and with a CO-modified tip (filled circles, at $V_{\text {bias }}=10 \mathrm{mV}$ ). Inset shows the tip height trace measured for $\mathrm{HH}$-porphycene with the $\mathrm{CO}$-modified tip. (c) Deformation of the tautomerization potential caused by the proximity of the tip and porphycene.

calculations also revealed the stable adsorption structure of the porphycene on $\operatorname{Ag}(110)$ and the potential energy surface of the cis $\leftrightarrow$ cis tautomerization. The planar macrocycle of porphycene in the gas phase was found to be deformed upon adsorption, but the cis configuration is stabilized by the strong interaction between the amine nitrogen atoms in the molecular cavity and the $\mathrm{Ag}$ atoms underneath. The calculated barrier of $0.064 \mathrm{eV}$ for the stepwise pathway was too large to be overcome at cryogenic temperatures, corroborating that the tautomerization is governed by tunneling. It was also found that the vibrational excitation of a skeletal mode via an inelastic electron tunneling process increases the tautomerization rate. Moreover, the tunneling dynamics could be manipulated by varying the tip-molecule distance (interaction) which changes the tunneling rate and eventually quenches the reaction through modification of the potential energy surface of the tautomerization. We believe that our results represent a benchmark experiment for examining a double hydrogen 
transfer via tunneling in multidimensional potential energy surfaces with a high level of theoretical simulations.

\section{ASSOCIATED CONTENT}

\section{S Supporting Information}

The Supporting Information is available free of charge on the ACS Publications website at DOI: 10.1021/jacs.7b06905.

Characterization of $\mathrm{HH}-, \mathrm{HD}$-, and DD-porphycene on the surface, $\mathrm{d} I / \mathrm{d} V$ spectra for $\mathrm{HH}-, \mathrm{DD}_{-}$, and $\mathrm{HD}$ porphycene on $\mathrm{Cu}(110)$ at $5 \mathrm{~K}$, voltage-dependent tautomerization yield (STM-action spectra) for $\mathrm{HH}$-, DD-, and HD-porphycene on $\mathrm{Cu}(110)$ at $5 \mathrm{~K}$, trace of tip height $(Z)$ measured for DD- and HD-porphycene on $\mathrm{Ag}(110)$ at $5 \mathrm{~K}$, current dependence of $\mathrm{HH}-$, HD-, and DD-porphycene on the $\mathrm{Ag}(110)$ surface, current dependence of the tautomerization rates measured on $\mathrm{Ag}(110)$ at $5 \mathrm{~K}$ for $\mathrm{HH}$-, $\mathrm{DD}$-, and $\mathrm{HD}$-porphycene, details of the density functional theory (DFT) calculations, calculated bucklings of the molecular skeleton $(\Delta B)$ potential energies $(E)$, zero-point-energy corrected energies $(\tilde{E})$, and imaginary vibrational energies $\left(i \hbar \omega_{1}\right)$, calculated energies of the isolated porphycene in the buckled geometries of the adsorbed porphycene in the cis configuration, transition state (TS), and the trans configuration, calculated potential energy differences for the cis $\rightarrow$ trans and the cis $\rightarrow$ TS transitions using different exchange-correlation energy functionals, minimum energy paths (MEPs) of the stepwise and concerted hydrogen transfer, MEPs and calculated structures on the $\mathrm{Cu}(110)$ and $\mathrm{Ag}(110)$ surfaces, additional references (PDF)

\section{AUTHOR INFORMATION}

\section{Corresponding Author}

*kuma@fhi-berlin.mpg.de

\section{ORCID $\odot$}

Takashi Kumagai: 0000-0001-7029-062X

\section{Notes}

The authors declare no competing financial interest.

\section{ACKNOWLEDGMENTS}

The authors thank Mariana Rossi and Martin Wolf for stimulating discussions. T.K. acknowledges the support of Morino Foundation for Molecular Science. M.P. acknowledges computer time allocated on ARCHER through the Materials Chemistry Consortium (EPSRC grant no. EP/L000202), on Polaris through N8 HPC (EPSRC grant no. EP/K000225/1), and on Chadwick at the University of Liverpool. J.W. acknowledges the grant from the Polish National Science Centre (DEC-2013/10/M/ST4/00069).

\section{REFERENCES}

(1) Hynes, J. T.; Klinman, J. P.; Limbach, H.-H.; Schowen, R. L. Hydrogen-Transfer Reactions; Wiley-VCH, 2007.

(2) Tuckerman, M. E.; Marx, D.; Klein, M. L.; Parrinello, M. Science 1997, 275, 817.

(3) Tuckerman, M. E.; Marx, D.; Parrinello, M. Nature 2002, 417, 925.

(4) Li, X.-Z.; Walker, B.; Michaelides, A. Proc. Natl. Acad. Sci. U. S. A. 2011, 108, 6369.
(5) Ceriotti, M.; Fang, W.; Kusalik, P. G.; McKenzie, R. H.; Michaelides, A.; Morales, M. A.; Markland, T. E. Chem. Rev. 2016, 116, 7529.

(6) Hund, F. Z. Phys. 1927, 43, 803.

(7) McMahon, R. J. Science 2003, 299, 833.

(8) Shannon, R. J.; Blitz, M. A.; Goddard, A.; Heard, D. E. Nat. Chem. 2013, 5, 745.

(9) Klinman, J. P.; Kohen, A. Annu. Rev. Biochem. 2013, 82, 471.

(10) Bell, R. P. The Tunneling Effect in Chemistry; Chapman and Hall, 1980.

(11) Lauhon, L. J.; Ho, W. Phys. Rev. Lett. 2000, 85, 4566.

(12) Kumagai, T.; Kaizu, M.; Hatta, S.; Okuyama, H.; Aruga, T.; Hamada, I.; Morikawa, Y. Phys. Rev. Lett. 2008, 100, 166101.

(13) Meng, X.; Guo, J.; Peng, J.; Chen, J.; Wang, Z.; Shi, J.-R.; Li, X.Z.; Wang, E.-G.; Jiang, Y. Nat. Phys. 2015, 11, 235.

(14) Kumagai, T.; Kaizu, M.; Okuyama, H.; Hatta, S.; Aruga, T.; Hamada, I.; Morikawa, Y. Phys. Rev. B: Condens. Matter Mater. Phys. 2009, 79, 035423.

(15) Kumagai, T.; Kaizu, M.; Okuyama, H.; Hatta, S.; Aruga, T.; Hamada, I.; Morikawa, Y. Phys. Rev. B: Condens. Matter Mater. Phys. 2010, 81, 045402.

(16) Guo, J.; Lü, J.-T.; Feng, Y.; Chen, J.; Peng, J.; Lin, Z.; Meng, X.; Wang, Z.; Li, X.-Z.; Wang, E.-G.; Jiang, Y. Science 2016, 352, 321.

(17) Ranea, V. A.; Michaelides, A.; Ramírez, R.; de Andres, P. L.; Vergés, J. A.; King, D. A. Phys. Rev. Lett. 2004, 92, 136104.

(18) Li, X.-Z.; Probert, M. I. J.; Alavi, A.; Michaelides, A. Phys. Rev. Lett. 2010, 104, 066102.

(19) Davidson, E. R. M.; Alavi, A.; Michaelides, A. Phys. Rev. B: Condens. Matter Mater. Phys. 2010, 81, 153410.

(20) Repp, J.; Fölsch, S.; Meyer, G.; Rieder, K.-H. Phys. Rev. Lett. 2003, 91, 206102.

(21) Stroscio, J. A.; Celotta, R. J. Science 2004, 306, 242.

(22) Heinrich, A. J.; Lutz, C. P.; Gupta, J. A.; Eigler, D. M. Science 2002, 298, 1381.

(23) Liljeroth, P.; Repp, J.; Meyer, G. Science 2007, 317, 1203.

(24) Sperl, A.; Kröger, J.; Berndt, R. Angew. Chem., Int. Ed. 2011, 50, 5294.

(25) Auwärter, W.; Seufert, K.; Bischoff, F.; Ecija, D.; Vijayaraghavan, S.; Joshi, S.; Klappenberger, F.; Samudrala, N.; Barth, J. V. Nat. Nanotechnol. 2012, 7, 41.

(26) Vogel, E.; Köcher, M.; Schmickler, H.; Lex, J. Angew. Chem., Int. Ed. Engl. 1986, 25, 257.

(27) Waluk, J. Chem. Rev. 2017, 117, 2447.

(28) Fita, P.; Grill, L.; Listkowski, A.; Piwonski, H.; Gawinkowski, S.; Pszona, M.; Sepiol, J.; Mengesha, E. T.; Kumagai, T.; Waluk, J. Phys. Chem. Chem. Phys. 2017, 19, 4921.

(29) Waluk, J. Acc. Chem. Res. 2006, 39, 945.

(30) Yoshikawa, T.; Sugawara, S.; Takayanagi, T.; Shiga, M.; Tachikawa, M. Chem. Phys. Lett. 2010, 496, 14.

(31) Vdovin, A.; Waluk, J.; Dick, B.; Slenczka, A. ChemPhysChem 2009, 10, 761.

(32) Ciąćka, P.; Fita, P.; Listkowski, A.; Radzewicz, C.; Waluk, J. J. Phys. Chem. Lett. 2016, 7, 283.

(33) Yoshikawa, T.; Sugawara, S.; Takayanagi, T.; Shiga, M.; Tachikawa, M. Chem. Phys. 2012, 394, 46.

(34) Kumagai, T.; Hanke, F.; Gawinkowski, S.; Sharp, J.; Kotsis, K.; Waluk, J.; Persson, M.; Grill, L. Phys. Rev. Lett. 2013, 111, 246101.

(35) Kumagai, T.; Hanke, F.; Gawinkowski, S.; Sharp, J.; Kotsis, K.; Waluk, J.; Persson, M.; Grill, L. Nat. Chem. 2014, 6, 41.

(36) Fita, P.; Urbańska, N.; Radzewicz, C.; Waluk, J. Z. Phys. Chem. 2008, 222, 1165.

(37) Kresse, G.; Furthmüller, J. Phys. Rev. B: Condens. Matter Mater Phys. 1996, 54, 11169.

(38) Kresse, G.; Joubert, D. Phys. Rev. B: Condens. Matter Mater. Phys. 1999, 59, 1758.

(39) Dion, M.; Rydberg, H.; Schröder, E.; Langreth, D. C.; Lundqvist, B. I. Phys. Rev. Lett. 2004, 92, 246401.

(40) Román-Pérez, G.; Soler, J. M. Phys. Rev. Lett. 2009, 103, 096102. 
(41) Klimeš, J.; Bowler, D. R; Michaelides, A. J. Phys.: Condens. Matter 2010, 22, 022201.

(42) Klimeš, J.; Bowler, D. R; Michaelides, A. Phys. Rev. B: Condens. Matter Mater. Phys. 2011, 83, 195131.

(43) Wellendorff, J.; Lundgaard, K. T.; Møgelhøj, A.; Petzold, V.; Landis, D. D.; Nørskov, J. K.; Bligaard, T.; Jacobsen, K. W. Phys. Rev. B: Condens. Matter Mater. Phys. 2012, 85, 235149.

(44) Berland, K.; Hyldgaard, P. Phys. Rev. B: Condens. Matter Mater. Phys. 2014, 89, 035412.

(45) Mills, G.; Jónsson, H.; Schenter, G. K. Surf. Sci. 1995, 324, 305.

(46) Henkelman, G.; Uberuaga, B. P.; Jónsson, H. A. J. Chem. Phys. 2000, 113, 9901.

(47) Limbach, H.-H.; Lopez, J. M.; Kohen, A. Philos. Trans. R. Soc., B 2006, 361, 1399.

(48) Limbach, H.-H. Hydrogen-Transfer Reactions; Wiley-VCH: Weinheim, 2007; Chapter 6, p 135 and references therein.

(49) Wehrle, B.; Limbach, H.-H. Chem. Phys. 1989, 136, 223.

(50) Klein, O.; Aguilar-Parrilla, F.; Lopez, J. M.; Jagerovic, N.; Elguero, J.; Limbach, H.-H. J. Am. Chem. Soc. 2004, 126, 11718.

(51) Lopez, J. M.; Männle, F.; Wawer, I.; Buntkowsky, G.; Limbach, H.-H. Phys. Chem. Chem. Phys. 2007, 9, 4498.

(52) Smedarchina, Z.; Shibl, M. F.; Kühn, O.; Fernández-Ramos, A. Chem. Phys. Lett. 2007, 436, 314.

(53) Ladenthin, J. N.; Frederiksen, T.; Persson, M.; Sharp, J. C.; Gawinkowski, S.; Waluk, J.; Kumagai, T. Nat. Chem. 2016, 8, 935. 